\title{
LOCALISATION DE LA PÊCHE, EFFECTIFS DE PÊCHEURS ET PRODUCTION DES ESPÈCES AMPHIHALINES DANS LES FLEUVES FRANÇAIS.
}

\section{G. CASTELNAUD}

CEMAGREF Bordeaux - Unité de Recherche Ressources Aquatiques Continentales 50, Avenue de Verdun, 33612 Cestas cedex, France.

Tel : 0557890803 - Fax : 0557890801 - E-mail : gerard.castelnaud@cemagref.fr

Reçu le 15 novembre 1999

Accepté le 07 septembre 2000
Received 15 November, 1999

Accepted 07 September, 2000

\section{RÉSUMÉ}

II n'existe pas de bilan récent sur la pêche des poissons et agnathes amphihalins en France prenant en compte l'ensemble des espèces, l'ensemble des catégories de pêcheurs et l'ensemble des zones exploitées.

Face à ce constat, nous avons analysé les résultats des études et des publications les plus récentes sur les pêches intérieures aux filets et aux engins ainsi que les résultats des suivis statistiques des captures régionaux ou nationaux, afin d'aboutir à une estimation plus actuelle et plus précise des effectifs de pêcheurs et des productions de la pêche des espèces amphihalines dans les fleuves français.

Tous les départements côtiers, sauf le Nord et le Pas-de-Calais, renferment des pêcheries d'anguilles au stade civelle surtout et aussi au stade jaune et argenté. Ces pêcheries sont essentiellement commerciales et beaucoup plus importantes dans le Golfe de Gascogne, alors que celles de saumon et truite de mer sont essentiellement sportives et se concentrent en cette qualité plutôt en Manche - Bretagne. Sur le bassin de la Loire la pêche du saumon est interdite et sur le bassin de la Garonne, celle des deux espèces de salmonidés l'est. Du fait de ces interdictions et bien que la pêche de l'alose feinte y soit limitée, le bassin de l'Adour vient en tête avec six espèces officiellement pêchées. On passe à cinq espèces dans le bassin de la Garonne puis à trois sur le bassin de la Loire, à deux sur le bassin de la Charente et du Rhône où l'on recherche une espèce différente d'alose. Sur les bassins du Rhin, de la Seine et de la Vilaine, on capture seulement l'anguille.

Nous avons dénombré, pour l'année de référence 1997, 1413 professionnels dont 448 professionnels en eau douce et 965 marins-pêcheurs, 1423 pêcheurs nonprofessionnels maritimes à pied, 7536 pêcheurs non-professionnels fluviaux à pied ou en bateau, 2974 pêcheurs aux lignes de saumon et 2030 pêcheurs aux lignes de truite de mer, recherchant les espèces amphihalines dans les cours d'eau français.

Au total, on obtient une production en poids de 1750 tonnes et un chiffre d'affaires de $462 \mathrm{MF}$ avec 410,5 tonnes et $410,5 \mathrm{MF}$ pour la civelle, 302 tonnes et $18,1 \mathrm{MF}$ pour languille, 581 tonnes et $8,7 \mathrm{MF}$ pour les aloses, 140 tonnes et $15,4 \mathrm{MF}$ pour la lamproie marine, 14,3 tonnes et $1,6 \mathrm{MF}$ pour les salmonidés et 303,5 tonnes pour $7,4 \mathrm{MF}$ pour le flet et le mulet. La production des pêcheurs autres que professionnels est prise en compte pour la civelle dans son ensemble, pour l'essentiel des aloses et pour les salmonidés qui ont la particularité d'être spécialement recherchés par les pêcheurs aux lignes. 
Le chiffre d'affaires de la pêche des amphihalins est dominé par l'espèce anguille, spécialement par le stade civelle qui en détermine à lui seul $89 \%$. Ainsi en 1997, la civelle passe au premier rang des ressources du Golfe de Gascogne avec une valeur 1,66 fois supérieure à celle de la sole.

Cette position hégémonique de l'anguille est discutée en regard de la viabilité de la pêche professionnelle dans les eaux intérieures françaises.

Mots-clés : espèces amphihalines, France, fleuves, géographie, effort de pêche nominal, production.

\section{FISHING LOCATION, FISHERMEN AND PRODUCTION OF MIGRATORY SPECIES FISHERIES IN THE FRENCH RIVERS.}

\section{ABSTRACT} rivers.

No recent statement exists concerning the migratory species fisheries in the French

To estimate the more precisely the number of fishermen and the productions, we gathered and analysed all the literature available and used the results of the regional and national statistical fisheries survey.

In all the French coastal departments, except Nord and Pas-de-Calais, we can find eel fisheries, particularly glass eel fisheries. They are more important along the Bay of Biscay and are mainly commercial fisheries. At the contrary the Salmonids fisheries are concentrated along the Brittany and the English Channel coast, and are mainly sportive fisheries.

In the Loire basin, the salmon fishing is banned and in the Garonne basin, the salmon fishing and the trout fishing are banned. According to these bans, the Adour basin is on the top with six migratory species fished, though the twaite shad fishing is limited. Five different migratory species are captured in the Garonne basin, three in the Loire basin, two in the Charente basin and in the Rhône basin, where a different specie of shad occur. In the Rhine basin, the Seine basin and the Vilaine basin, only eel is captured.

We counted a total of 1413 professional fishermen, divided in 448 river professional fishermen, and 965 marine professional fishermen, a total of 1423 marine nonprofessional fishermen, 7536 river non-professional fishermen, 2974 sportive salmon fishermen and 2030 sportive sea trout fishermen, who capture migratory species in French rivers.

The total production is 1750 tons with a turn-over of 462 millions FF ; this production is composed of 410,5 tons and 410,5 millions FF for the glass eel, 302 tons and 18,1 millions FF for the eel, 581 tons and 8,7 millions de FF for the shad, 140 tons and 15,4 millions FF for the lamprey, 14,3 tons and 1,6 million FF for the salmonids species, 303,5 tons and 7,4 millions FF for flounder and mullet. The production of the nonprofessional fishermen is evaluated and included for the glass eels, for the main part of shad and for the Salmonids which are especially fished by anglers.

The turn-over of the migratory species fisheries is dominated by eel, especially the glass eel stage which provide $89 \%$. In 1997, the glass eel is on the top of the fisheries resources of the Bay of Biscay with a value equal to $1,66 \%$ of the value of the sole. 
The particular position of glass eel among the migratory species is discussed in connection with the viability of professional fisheries in the French inland waters.

Key-words : migratory species, France, rivers, location, fishing effort, production.

\section{INTRODUCTION}

II n'existe pas de bilan récent sur la pêche des poissons et agnathes amphihalins en France prenant en compte l'ensemble des espèces, l'ensemble des catégories de pêcheurs et l'ensemble des zones exploitées.

Les quelques travaux disponibles ont raisonné par entités biogéographiques où l'essentiel des espèces, mais pas toutes, étaient des migrateurs amphihalins. ANONYME (1987), dans un travail d'archives très fouillé, a analysé les statistiques des pêches maritimes disponibles pour les principales espèces amphihalines de 1896 à 1986 dans les estuaires, et a tenté de les relier à des phénomènes économiques et sociaux : évolution du nombre de pêcheurs, des prix de vente des espèces et du commerce extérieur de la France en poissons d'eau douce. L'état établi par CASTELNAUD et de VERDILHAC (1982) concernait aussi les estuaires et les zones mixtes des grands fleuves, avec un "blanc " pour le Rhône ; 1500 professionnels et 4000 amateurs environ dégageaient un chiffre d'affaires de $130 \mathrm{MF}$, pour une production de 3600 tonnes en 1981.

ELIE (1986), dans son évaluation pour l'année de référence 1985, prenait lui aussi en compte essentiellement les estuaires et les zones mixtes des fleuves du littoral de la façade Manche - Atlantique ; il arrivait à une production de 1650 tonnes de poissons et d'agnathes amphihalins à laquelle il ajoutait une production d'anguilles de 1700 tonnes pour les lagunes méditerranéennes. Entre temps, l'intervention du CEMAGREF au sein du "Groupe Evaluation des Stocks " réuni par I'ISTPM" en 1982-1983, montrait, à la stupéfaction de tous, que l'anguille au stade civelle se plaçait sur le plan économique à la $4^{\natural}$ place parmi les espèces marines capturées dans le Golfe de Gascogne (ISTPM, 1983 ; ELIE et RIGAUD, 1984).

Par la suite, les chiffres globaux mis à l'appui de l'importance des espèces amphihalines ont été dérivés des précédents et des états annuels de délivrance de licences aux marins-pêcheurs par la CIPE ${ }^{2}$ (ELIE et RIGAUD, 1984) puis renforcés par ceux qui se rapportaient à la pêche professionnelle en eau douce (CASTELNAUD et BABIN, 1992 ; BABIN, 1993) : 1000 à 1200 marins-pêcheurs, 400 à 500 entreprises de pềche fluviale, 4 à 6000 amateurs, 140 à 150 MF de chiffre d'affaires (ELIE, 1992, 1993). Le panorama exhaustif de la pêche et de l'aquaculture sur le territoire national qu'a dressé W.A. DILL (1993) pour la FAO-CECPI ${ }^{3}$ au début des années 90 conduit à des chiffres parcellaires et le plus souvent sous-évalués en ce qui concerne les amphihalins, malgré une recherche documentaire poussée.

Face à ce constat, il apparaît que les résultats des études et des publications les plus récentes sur les pêches aux filets et aux engins dans les eaux intérieures françaises ainsi que les résultats des suivis statistiques des captures disponibles au niveau régional et national, peuvent être utilisés dans une tentative d'estimation des effectifs de pêcheurs et des productions plus actuelle et plus précise de la pêche des espèces amphihalines.

\footnotetext{
"Institut Scientifique et Technique des Pêches Maritimes, devenu IFREMER

${ }^{2}$ Commission des Poissons Migrateurs et des Estuaires, organisme professionnel national des Pêches Maritimes et des Elevages Marins.

Commission Européenne Consultative pour les Pêches dans les eaux intérieures de la FAO.
} 
C'est le travail que nous nous proposons d'effectuer dans cette publication, afin qu'à l'aube $\mathrm{du} x \mathrm{x}^{\mathrm{e}}$ siècle, dans l'attente des résultats futurs des suivis statistiques officiels mis en place progressivement par le $\mathrm{CSP}^{4}$ et le CRTS ${ }^{5}$, les scientifiques et les différents acteurs de la gestion des espèces amphihalines puissent disposer d'un bilan de référence. $\mathrm{C}_{e}$ bilan concernera la pêche aux filets et aux engins et en partie la pêche aux lignes des espèces amphihalines sur le domaine public fluvial, c'est-à-dire sur l'ensemble des fleuves français jusqu'à l'embouchure, lagunes méditerranéennes exclues. II sera établi pour l'année de référence 1997.

\section{MATÉRIEL ET MÉTHODE}

\section{Espèces concernées}

Toutes les espèces amphihalines présentes sur le territoire national, sauf l'esturgeon européen Acipenser sturio, entièrement protégé, font actuellement l'objet de pêches et sont donc prises en compte dans cette śvaluation. Elles se divisent en deux groupes. Le premier groupe comprend les espèces suivies de façon prioritaire du fait de leur grand intérêt halieutique et pour lesquelles il existe des données plus ou moins nombreuses et précises : alose vraie (Alosa alosa), lamproie marine (Petromyzon marinus), anguille (Anguilla anguilla) stades civelle, jaune et argenté, saumon (Salmo salar) et truite de mer (Salmo trutta). Le second groupe comprend les espèces suivies de façon secondaire, du fait de leur moindre intérêt halieutique et économique ou d'une abondance apparente faible et pour lesquelles les données sont rares : mulet (Liza ramada), flet (Platichthys flesus), aloses feintes (Alosa fallax fallax et Alosa fallax rhodanensis) et lamproie fluviatile (Lampetra fluviatilis).

\section{Délimitation géographique de la pêche des différentes espèces}

La pêche des espèces amphihalines est délimitée de façon globale par département, à partir de la superposition :

- de la carte de répartition des pêcheurs professionnels qui recherchent les espèces amphihalines, (CASTELNAUD et BABIN 1992, BABIN 1993, CASTELNAUD et al., 1994);

- de la carte de répartition des captures de salmonidés en 1997 (PORCHER, 1998 et FOURNEL et al. 1998);

- de la carte de présence de l'anguille (ALLARDI et KEITH, 1991 ; KEITH, 1998; CHANCEREL, 1994);

- de la carte de répartition en 1997 des pêcheurs amateurs aux filets et aux engins qui sont susceptibles d'en capturer, établie par le CSP (CHANGEUX, com. pers.).

On suppose que dans les départements des hauts de bassins où les anguilles sont recensées, les pêcheurs amateurs qui ont droit généralement à des nasses et à des lignes de fond, capturent cette espèce.

Cette pêche des espèces amphihalines est ensuite dissociée de façon plus précise par espèce et par zone de pêche à partir des informations contenues dans CASTELNAUD et BABIN (1992), CASTELNAUD et al. (1994), PORCHER (1998) ef FOURNEL et al. (1998) et des informations obtenues auprès des experts scientifiques, des administrations et des représentants des pêcheurs. Les zones de pêche représentent un ou plusieurs bassins fluviaux qui sont découpés en secteurs, fonction des limites administratives et des

\footnotetext{
"Conseil Supérieur de la Pêche

sentre Régional de Traitement Statistique des pêches maritimes.
} 
données disponibles sur les effectifs de pêcheurs et les productions. Le bassin de la Garonne par exemple, est découpé en un secteur aval appelé Gironde qui comprend l'estuaire et les zones mixtes de Garonne et Dordogne et en un secteur amont appelé Garonne-Dordogne.

\section{Les effectifs et les productions}

Les effectifs de pêcheurs professionnels maritimes et fluviaux sont établis à partir des résultats de CASTELNAUD et al. (1994), à partir des résultats de l'enquête récente de CHAMPION (1999) confrontés à ceux de l'enquête de CASTELNAUD et BABIN (1992). Des ajouts et corrections sont effectués à partir des données de suivi des captures de 1997 de CASTELNAUD et al. (1999) pour la Gironde, des données de suivi statistiques de 1997 de PROUZET et al. (1998) et de CUENDE (1998) pour le bassin de l'Adour, des informations récentes provenant de la CNAPPED 6 (BOISNEAU, com. pers.) et de la CIPE (GUERNALEC, com. pers.).

Du fait que les pêcheurs aux filets et aux engins autres que professionnels ne sont pas seulement des amateurs qui ne commercialisent pas leurs prises, nous les regrouperons sous l'appellation "pêcheurs non-professionnels " comme dans CASTELNAUD et al. (1994). Lorsqu'on ne dispose pas d'informations particulières, on estime que les effectifs de pêcheurs recherchant les espèces amphihalines correspondent aux effectifs totaux de pêcheurs obtenus pour chaque secteur de pêche circonscrit.

Les effectifs de pêcheurs non-professionnels maritimes à pied correspondent aux pêcheurs non-professionnels maritimes à pied de civelles dénombrés en 1989 par CASTELNAUD et al. (1994) sauf pour le bassin de l'Adour et les courants landais où le chiffre a été actualisé en 1997 grâce aux données de PROUZET et al. (1998). Les effectifs de pêcheurs non-professionnels fluviaux aux filets et aux engins sont estimés sur la base des licences de pêche amateur délivrées en 1997 et enregistrées par le CSP (CHANGEUX, com. pers.) et pour la Gironde en 1997 sur la base des dénombrements effectués par CASTELNAUD et al. (1999). Les effectifs de pêcheurs de loisir aux lignes recherchant les salmonidés sont trouvés dans PORCHER (1998) et FOURNEL et al. (1998).

Les chiffres de production en poids proviennent essentiellement de CASTELNAUD et al. (1994) pour la pêche de la civelle en 1989, de BABIN (1993) pour la pêche de l'ensemble des espèces en 1989 par les professionnels fluviaux, de PORCHER (1998) et FOURNEL et al. (1998) pour la pêche des salmonidés en 1997 par les pêcheurs aux lignes et les professionnels. Ces chiffres sont complétés d'une part par ceux de GUERAULT et al. (1994) pour les captures en 1989 de certaines espèces par les marinspêcheurs, de CASTELNAUD et al. (1999) pour la pêche professionnelle et nonprofessionnelle de toutes les espèces autres que les salmonidés dans la Gironde, la Garonne et la Dordogne (moyenne 1990 - 1997), de PROUZET et al. (1998) et CUENDE (1998) pour la pêche professionnelle et non-professionnelle de toutes les espèces sur le bassin de l'Adour en 1997 (moyenne 1987 - 1997). Ces chiffres sont complétés d'autre part par ceux de ROBION et ADAM (1998) pour la pêche professionnelle de l'anguille dans le lac de Grand-Lieu en 1997, du CRTS (GRANDPIERRE, com. pers.) dans la Vie, le Lay et la Sèvre Niortaise, de CUENDE (1998, com. pers.) dans le Rhin en 1997, du CSP (CHANGEUX com. pers.) dans le Rhône et dans la Seine en 1997, de LE CORRE (1999) pour la pêche professionnelle de l'alose dans le Rhône.

Les poids de salmonidés capturés par les pêcheurs aux lignes ont été calculés à partir du poids moyen et de l'effectif total indiqués par FOURNEL et al. (1998) pour les

Coordination Nationale de la Pêche Professionnelle en Eau Douce 
truites de mer et à partir des poids moyens et des effectifs par classes d'âge indiqués par PORCHER (1998) pour les saumons.

Du fait du caractère très artisanal de la pêche dans les eaux intérieures françaises, les espèces variées sont commercialisées sur des marchés locaux, à partir de circuits courts ; la vente directe est très développée et les intermédiaires sont des mareyeurs, poissonniers, restaurateurs essentiellement. II n'existe pas de système d'enregistrement officiel des prix pratiqués comme en criée pour les poissons de mer et encore moins de mercuriale.

Afin d'estimer un chiffre d'affaires de la pêche des espèces amphihalines dans les fleuves français, nous avons déterminé comme BABIN (1993) un prix moyen de vente en 1997 par le pêcheur pour chaque espèce au niveau national à partir des informations fournies par CASTELNAUD et al. (1999), CHAMPION (1999), PROUZET et al. (1998), CUENDE (1998), par les représentants des pêcheurs (BOISNEAU, com. pers. ; GUERNALEC, com. pers.), les pêcheurs eux-mêmes et certains mareyeurs.

Les prix de vente de ces espèces sont assez semblables d'une région à l'autre. Pour la civelle, un prix de vente moyen de la campagne 1996 - 1997 a été obtenu auprès de la CIPE (GUERNALEC, com. pers.). On retient pour les aloses, le prix moyen de vente de l'alose vraie en Gironde, du fait de l'importance de la production de cette espèce dans cette région par rapport aux deux autres espèces d'aloses feintes et aux autres régions. Le saumon et la truite de mer ne sont déclarés à la vente que dans le bassin de l'Adour. Nous appliquerons donc le prix moyen pratiqué en 1997 dans cette région à l'ensemble de la production enregistrée dans les fleuves français. On calcule pour les autres espèces, un prix de vente moyen sur les principales zones de production, c'est-à-dire la Loire aval et la Gironde pour la lamproie et le flet, la Loire aval, le lac de Grand-Lieu et la Gironde pour l'anguille sub-adulte, le Rhône, la Loire aval et la Gironde pour le mulet.

\section{RÉSULTATS ET DISCUSSION}

\section{Localisation de la pêche}

La Figure 1 montre les départements où des pêcheurs professionnels, nonprofessionnels et de loisir capturent des espèces amphihalines de façon sûre et les départements où des pêcheurs non-professionnels capturent, certainement en amateurs vrais, des espèces amphihalines de façon très probable: Drôme, Isère, Mayenne, Indre, Puy-de-Dôme, Tarn-et-Garonne, Tarn, Haute-Garonne, Charente.

Dans les départements du Cantal et de Lozère, ni les pêcheurs amateurs, ni l'anguille ne sont présents et dans les départements de Corrèze, de Savoie et de HauteSavoie, qui abritent des pêcheurs aux filets et aux engins, soit ceux-ci ne recherchent pas l'anguille, soit celle-ci n'y est pas inventoriée.

Tous les départements côtiers, sauf le Nord et le Pas-de-Calais (Figure 1) renferment des pêcheries d'anguilles au stade civelle surtout et aussi au stade jaune et argenté. Ces pêcheries sont essentiellement commerciales et beaucoup plus importantes dans le Golfe de Gascogne, alors que celles de salmonidés sont essentiellement sportives et se concentrent en cette qualité plutôt en Manche-Bretagne : Somme, Seine-Maritime, Eure, Calvados, Manche, Ille-et-Vilaine, Côtes d'Armor, Finistère, Morbihan. Les pêcheries commerciales de salmonidés se trouvent dans les départements des Landes et des Pyrénées-Atlantiques. 


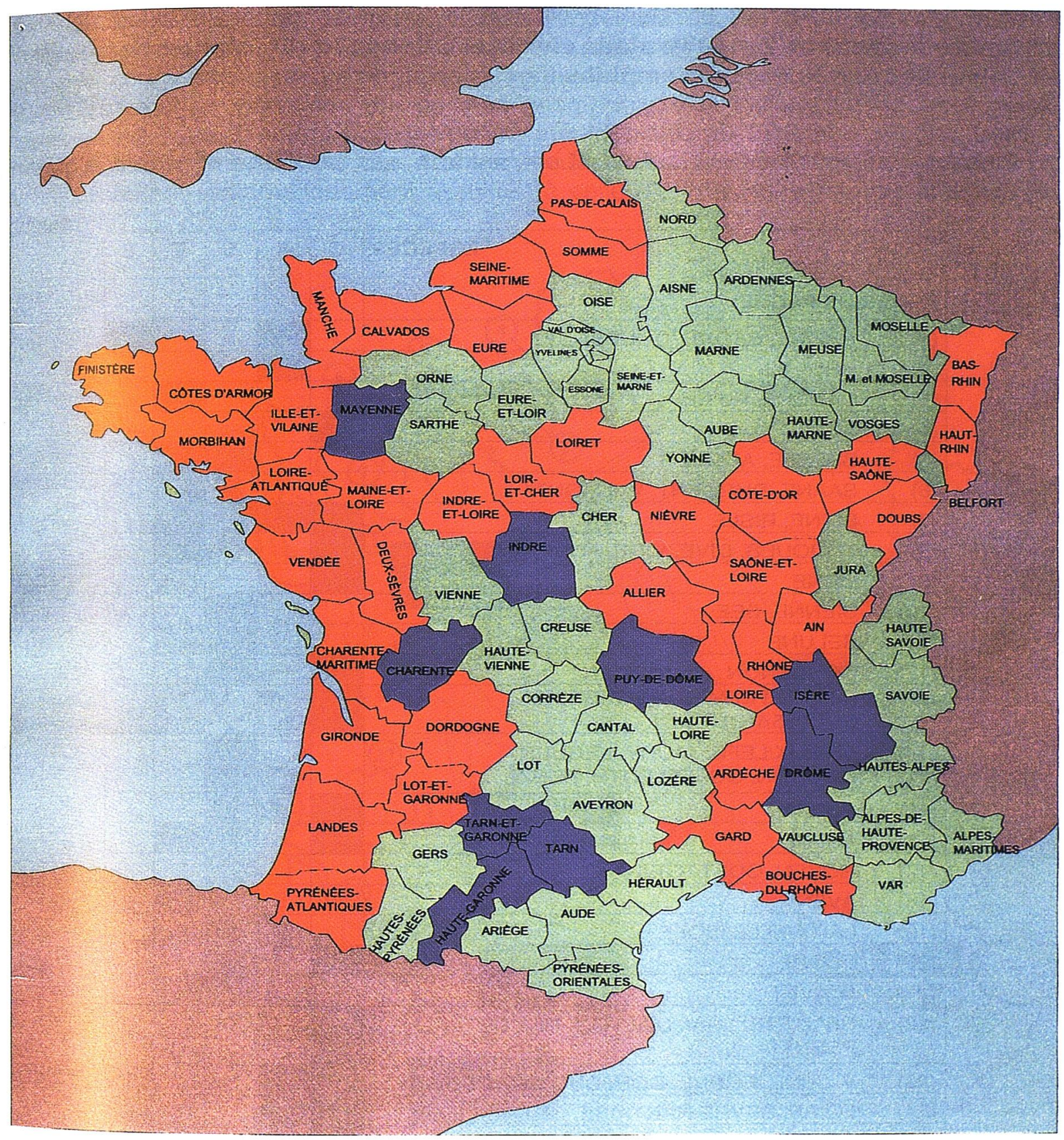

Présence de pêcheurs toutes catégories capturant des espèces amphihalines

Prćsence de pêcheurs amateurs aux filets et aux engins susceptibles de capturer des espèces amphihalines

\section{Figure 1}

Répalition par département des pêcheurs d'espèces amphihalines.

Figure :

Distribution of migratory species fishermen in the french departments. 


\section{Tableau I}

Zones de pêche de l'anguille stade civelle et sub-adulte, du saumon, de la truite de mer et des autres espèces amphihalines.

\section{Table I}

Fishing areas of glass eel, subadult eel, salmon, sea trout and of others migratory species.

\begin{tabular}{|c|c|c|c|c|c|c|}
\hline ZONES & ESPECES & $\mathrm{c}$ & $\mathbf{A}$ & $\mathbf{s}$ & $\mathbf{T}$ & $\mathbf{E}$ \\
\hline (1) & RHONE, SAONE, DOUBS & & & & & \\
\hline (2) & RHIN, ILL & & & & & \\
\hline (3) & CANCHE, AUTIE & & & & & \\
\hline (4) & SOMME & & & & & \\
\hline (5) & BRESLE, ARQUES & & & & & \\
\hline (6) & SAANE, DURDENT, VALMONT & & & & & \\
\hline (7) & SEINE, RISLE & & & & & \\
\hline (8) & TOUQUES, DIVE, VIRE, AURE & & & & & \\
\hline (9) & ORNE & & & & & \\
\hline$(10)$ & SIENNE, SEE & & & & & \\
\hline (11) & SELUNE & & & & & \\
\hline$(12)$ & COUESNON & & & & & \\
\hline (13) & $\begin{array}{l}\text { RANCE, FREMUR, ARGUENON, } \\
\text { GOUESSAN, GOUET }\end{array}$ & & & & & \\
\hline (14) & $\begin{array}{l}\text { TRIEUX/LEFF, JAUDY, LEGUER, } \\
\text { DOURON, QUEFFLEUTH, PENZE }\end{array}$ & & & & & \\
\hline$(15)$ & ELORN & & & & & \\
\hline$(16)$ & AULNE & & & & & \\
\hline$(17)$ & GOYEN, ODET/STEIR/JET & & & & & \\
\hline$(18)$ & AVEN & & & & & \\
\hline (19) & LAITA/ELLE/ISOLE & & & & & \\
\hline$(20)$ & SCORF & & & & & \\
\hline$(21)$ & BLAVET & & & & & \\
\hline (22) & $\begin{array}{l}\text { RIV.D'ETEL, RIV. D'AURAY, RIV. DE } \\
\text { PERNEF }\end{array}$ & & & & & \\
\hline$(23)$ & VILAINE, ERDRE, DON, OUST, CHERE & & & & & \\
\hline (24) & LOIRE, SEVRE-NANTAISE, GRAND-LIEU & & 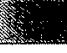 & & & \\
\hline$(25)$ & BAIE DE BOURGNEUF, VIE & & & & & \\
\hline$(26)$ & LAY & & & & & \\
\hline$(27)$ & SEVRE-NIORTAISE & & Xing & & & \\
\hline$(28)$ & CHARENTE, SEUDRE* & & & & & \\
\hline$(29)$ & GIRONDE, GARONNE, DORDOGNE, ISLE & & (2) & & & \\
\hline$(30)$ & CANAL DE LEGE & & & & & \\
\hline$(31)$ & $\begin{array}{l}\text { COURANTS DE MIMIZAN, CONTIS, } \\
\text { HUCHET, VIEUX-BOUCAU }\end{array}$ & & & & & \\
\hline$(32)$ & ADOUR, NIVE, BIDOUZE, GAVES & & & & & \\
\hline C & CIVELLE & & & & & \\
\hline A & ANGUILLE & & & & & \\
\hline $\mathbf{S}$ & SAUMON & & & & & \\
\hline $\mathrm{T}$ & TRUITE DE MER & & & & & \\
\hline$E$ & AUTRES ĖSPECES & & & & & \\
\hline * & SEUDRE : CIVELLE UNIQUEMENT & & & & & \\
\hline
\end{tabular}


Les cours d'eau qui se trouvent dans l'ensemble de ces départements, où sont enregistrées des captures de civelle, d'anguille jaune ou argentée, de saumon ou de truite de mer et des autres espèces amphihalines sont rassemblés par zones au Tableau 1. La Figure 2 permet de visualiser leur position géographique et indique toutes les espèces nobles recherchées dans les six bassins fluviaux identifiés au Tableau I : Rhône, Vilaine, Loire, Charente, Garonne, Adour. On remarque que la capture des autres espèces amphihalines est systématiquement associée à celle d'un ou plusieurs stades d'Anguilla anguilla.

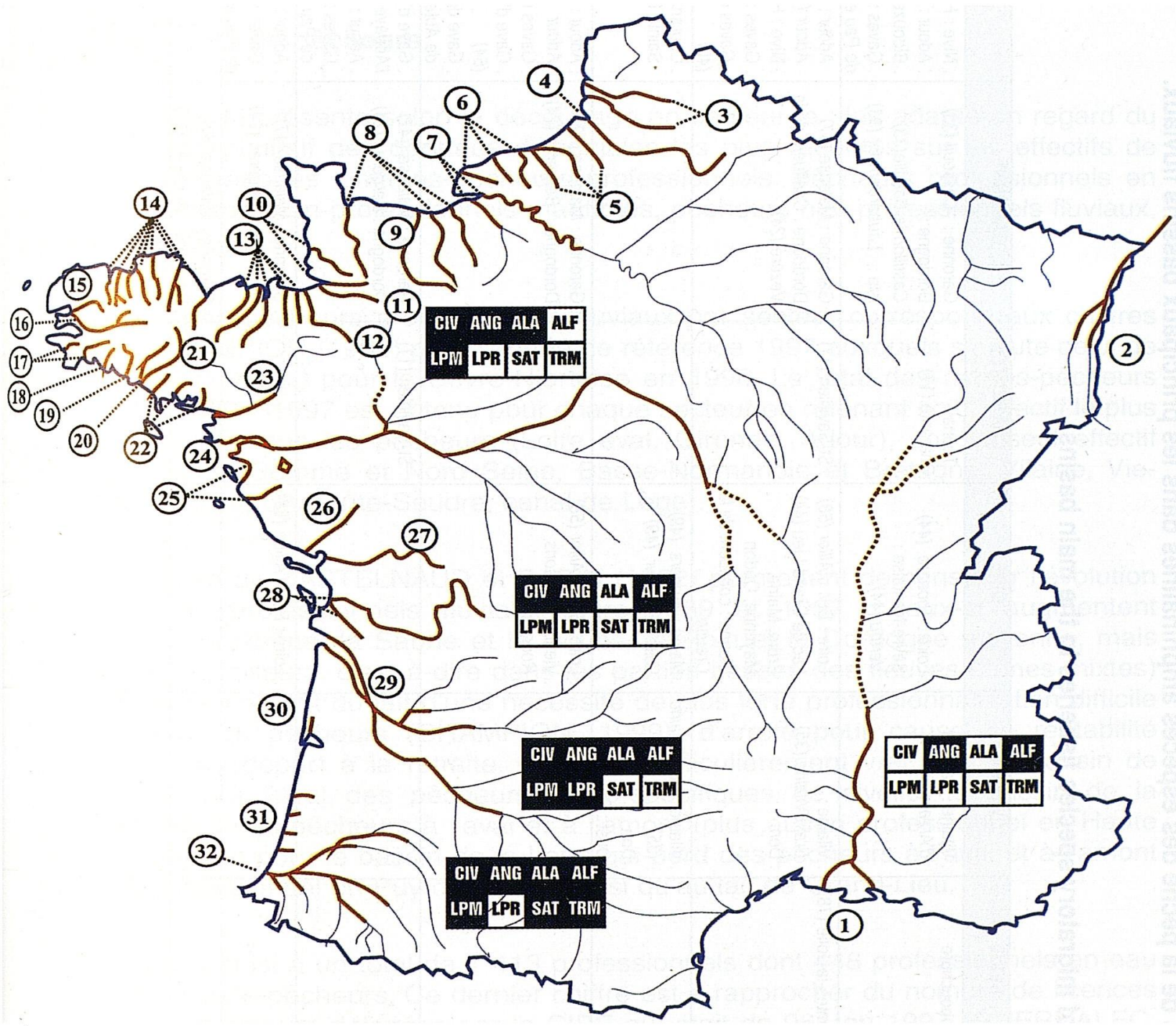

Figure 2

Carte de localisation des zones de pêche et des cours d'eau, avec les différentes espèces amphihalines nobles recherchées dans les grands bassins.

Figure 2

Map fishing areas and rivers with the different important migratory species fishec in the large basins.

Le Tableau II précise dans ces bassins ainsi que dans le bassin de la Seine, les cours d'eau et les parties de cours d'eau où se pratique la pêche des amphihalins. Les limites indiquées sont celles au delà desquelles, vraisemblablement, il n'est plus capturé d'espèces amphihalines, sauf peut-être l'anguille (Figures 1 et 2). 


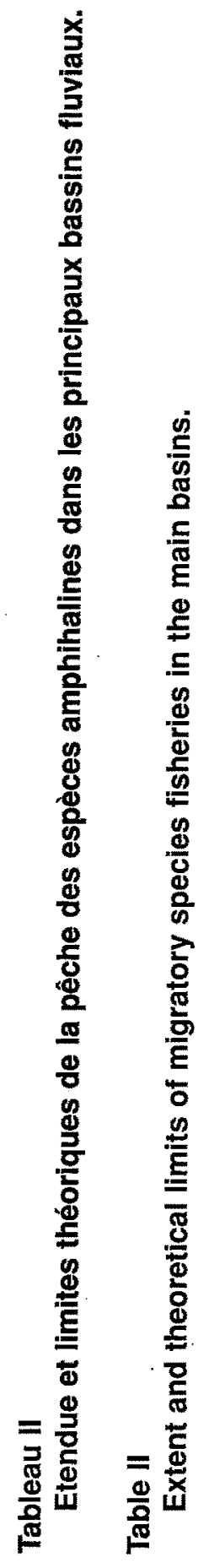

\begin{tabular}{|c|c|c|c|c|c|c|c|c|c|}
\hline 产 & 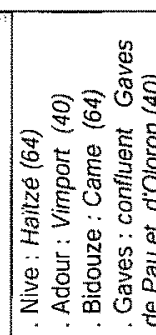 & 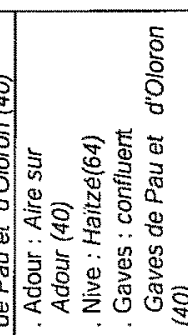 & 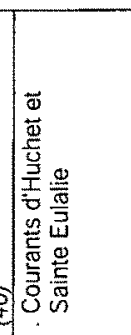 & 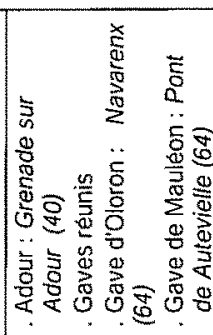 & 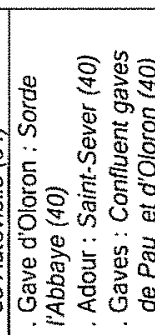 & 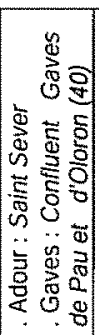 & & 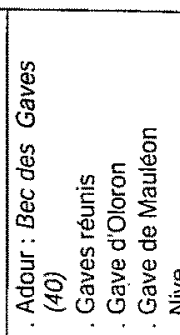 & 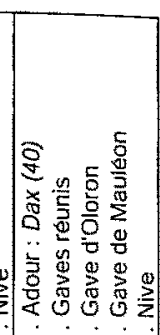 \\
\hline $\begin{array}{l}\frac{8}{8} \\
\\
0\end{array}$ & 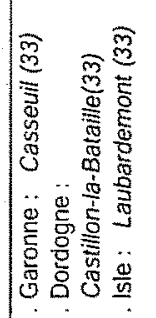 & 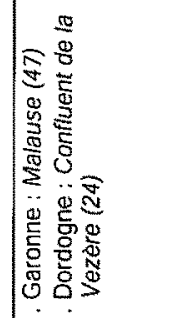 & & 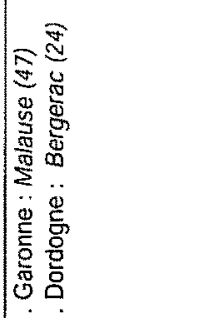 & 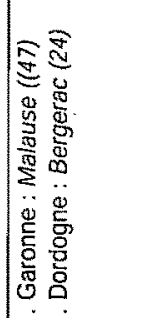 & 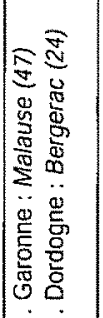 & 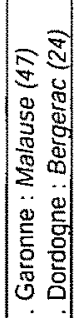 & & \\
\hline & 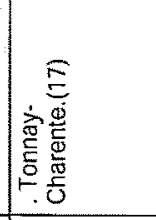 & 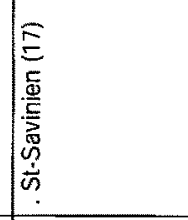 & & & 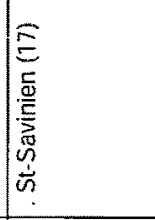 & & 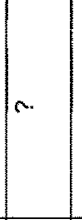 & & \\
\hline$\frac{u t}{\underline{\alpha}}$ & 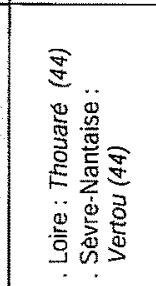 & 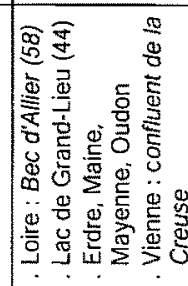 & 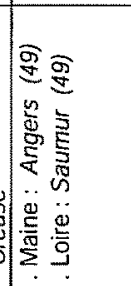 & 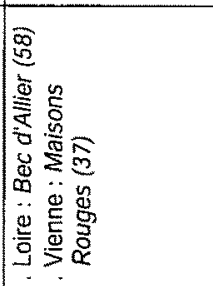 & & 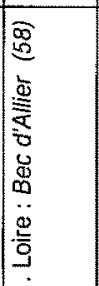 & $n$ & & \\
\hline Ue & 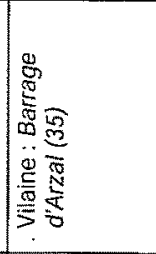 & 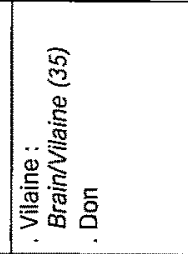 & 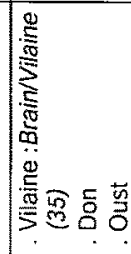 & & & & & & \\
\hline$\leq$ & 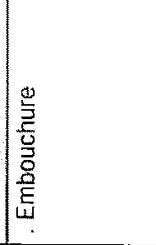 & 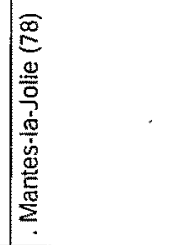 & & & & & & & \\
\hline & & 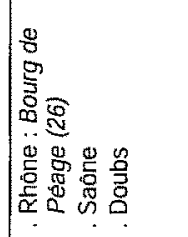 & 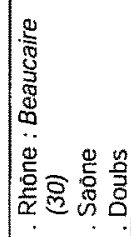 & & 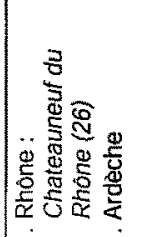 & & & & \\
\hline & 岂 & 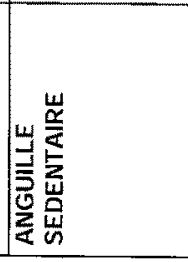 & 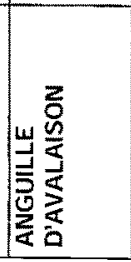 & 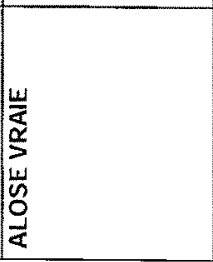 & 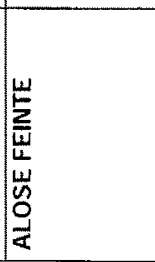 & 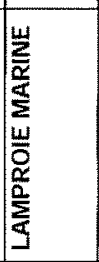 & 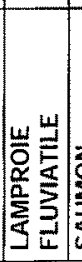 & 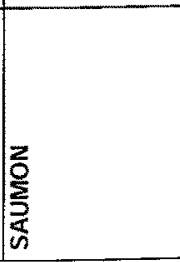 & 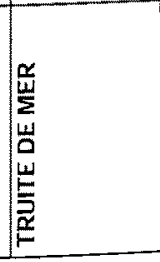 \\
\hline
\end{tabular}


Il faut remarquer que sur le bassin de la Loire la pêche du saumon est interdite et que sur le bassin de la Garonne, celle des deux espèces de salmonidés l'est. Du fait de ces interdictions et bien que la pêche de l'alose feinte y soit limitée, le bassin de l'Adour vient en tête pour le nombre d'espèces nobles officiellement pêchées. De six espèces on passe donc à cinq dans le bassin de la Garonne (Figure 2) puis à trois sur le bassin de la Loire, à deux sur le bassin de la Charente et du Rhône où l'on recherche une espèce différente d'alose. Sur les bassins du Rhin, de la Seine et de la Vilaine, on capture seulement l'anguille.

\section{Les effectifs de pêcheurs}

Le Tableau III présente selon le découpage en secteur le plus adapté en regard du Tableau 1, le récapitulatif des données disponibles les plus récentes sur les effectifs de pêcheurs, par catégories : marins-pêcheurs professionnels, pêcheurs professionnels en eau douce, pêcheurs non-professionnels maritimes, pêcheurs non-professionnels fluviaux, pêcheurs aux lignes.

Le total des pêcheurs professionnels fluviaux par secteur correspond aux chiffres produits par CHAMPION (1999) pour l'année de référence 1997 auxquels s'ajoute celui de BOISNEAU (com. pers.) pour la Sèvre-Niortaise en 1998. Le total des marins-pêcheurs sur la période 1989 - 1997 est obtenu pour chaque secteur en retenant soit l'effectif le plus actuel qui englobe tous les pêcheurs (Loire aval, Gironde, Adour), soit le seul effectif disponible (Rhône, Somme et Nord Seine, Basse-Normandie et Bretagne, Vilaine, VieLay-Sèvre Niortaise, Charente-Seudre, canal de Lège).

Les chiffres de CASTELNAUD et BABIN (1992) permettent de constater l'évolution des effectifs de professionnels fluviaux entre 1989 et 1997 : ceux-ci augmentent légèrement sur le Rhône, la Saône et le Doubs, le Rhin et la Dordogne moyenne, mais diminuent partout ailleurs, c'est-à-dire dans les parties basses des fleuves (zones mixtes) et dans les parties amont du fait d'une nécessité de plus forte professionnalisation difficile à assumer par les pêcheurs (CHAMPION, 1999), d'arrêts pour cause de rentabilité insuffisante ou de départ à la retraite. Ceci est particulièrement vrai pour le bassin de l'Adour qui perd à l'aval des pêcheurs mono-spécifiques de civelle, le bassin de la Garonne qui perd des pêcheurs à l'aval et à l'amont (plus aucun professionnel en Haute Garonne et Tarn) et pour le bassin de la Loíre qui perd des pêcheurs à l'aval et à l'amont (plus aucun professionnel en Puy de Dôme) ainsi qu'au lac de Grand-Lieu.

On aboutit ainsi à un total de 1413 professionnels dont 448 professionnels en eau douce et 965 marins-pêcheurs. Ce dernier chiffre est à rapprocher du nombre de licences de pêche aux migrateurs délivrées par la CIPE qui était de 961 en 1997 (GUERNALEC, com. pers.). Les origines diverses et le décalage dans le temps des chiffres de notre évaluation suffisent à expliquer la légère différence constatée. Mais il y a aussi des incertitudes entre la délivrance de licences et les pratiques réelles; par exemple, les pêcheurs d'anguille en mer côtière ne prennent pas forcément de licence. AUBRUN (1986) eI DESAUNAY et AUBRUN (1988) en dénombraient environ 88 en 1986.

If est difficile de rapporter ces effectifs à chaque espèce cible. Cependant, la majorité de ces pêcheurs capturent des anguilles aux différents stades. Compte tenu des seules évaluations actualisées de l'effort de pêche nominal que nous possédons, c'est-àdire celles effectuées sur la Gironde (CASTELNAUD et al., 1999) et sur l'Adour (PROUZET et al., 1998) nous retiendrons pour l'année de référence 1997, les chiffres suivants: 


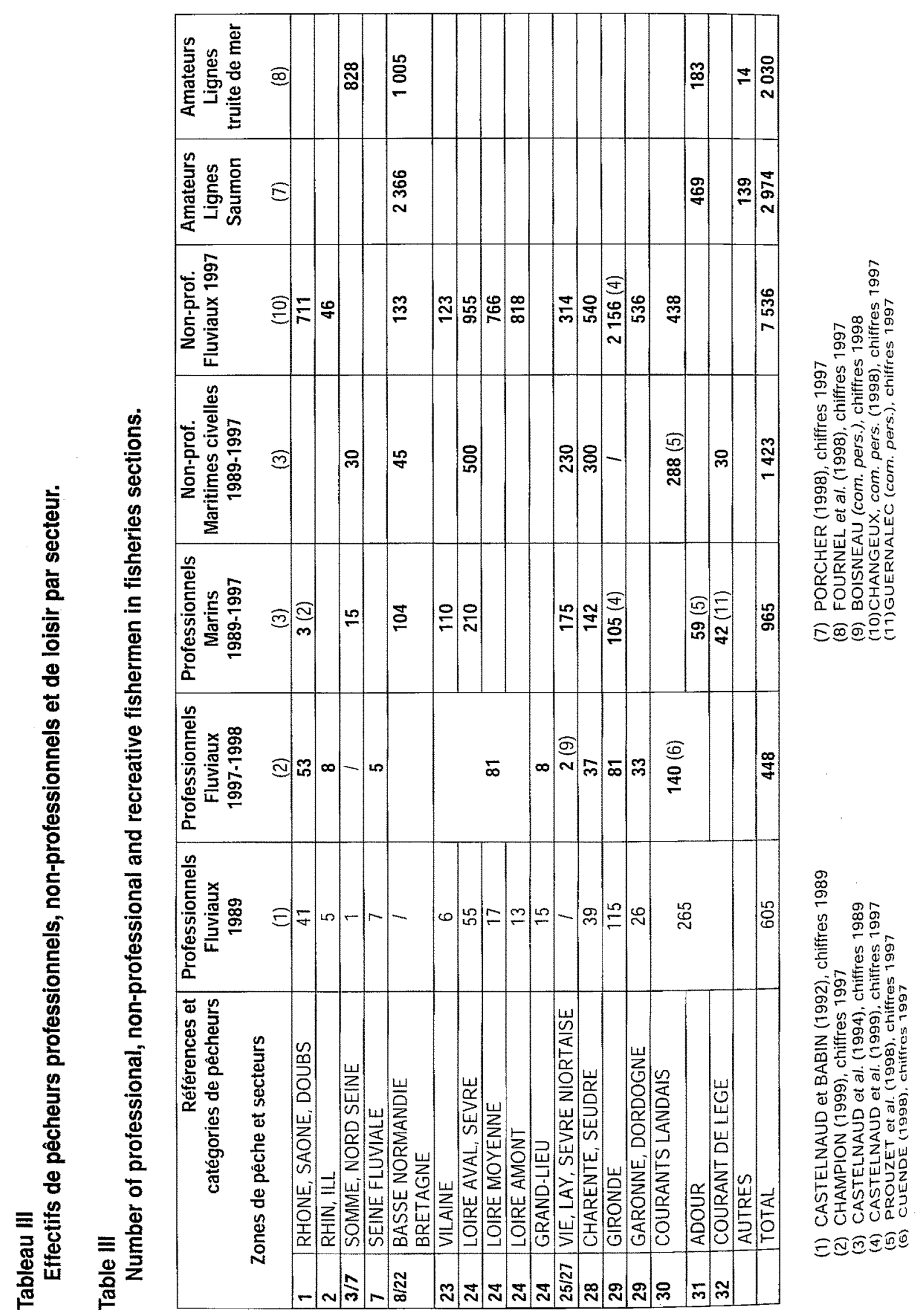


- la civelle est recherchée par 300 professionnels fluviaux et 930 marins-pêcheurs environ; l'anguille sédentaire ou d'avalaison est recherchée par 220 professionnels fluviaux dont 150 pêchent aussi la civelle et 110 marins-pêcheurs environ qui pêchent pratiquement tous aussi la civelle; l'anguille tous stades confondus mobilise 1300 professionnels environ;

- les aloses vraie et feinte sont recherchées essentiellement sur les bassins de la Loire, de la Garonne, de l'Adour, du Rhône et de la Charente, et peuvent concerner environ 150 professionnels fluviaux et 130 marins-pêcheurs ;

- la lamproie marine absente du Rhône, peut concerner un effectif un peu moindre de pêcheurs professionnels, soit 260 environ;

- les salmonidés pourraient concerner une cinquantaine de pêcheurs professionnels ou plus avec les captures accidentelles.

A titre de comparaison, BABIN (1993) dénombrait en 1989 parmi les professionnels fluviaux, 237 pêcheurs de civelles, 240 pêcheurs d'anguille, 186 pêcheurs de lamproie marine, 178 pêcheurs d'alose vraie, 53 pêcheurs de saumon.

On aboutit au Tableau III à un total de 1423 pêcheurs non-professionnels maritimes à pied, 7536 pêcheurs non-professionnels fluviaux à pied ou en bateau, 2974 pêcheurs aux lignes de saumon et 2030 pêcheurs aux lignes de truite de mer.

Nous n'avons aucune information sur les pêcheurs non-professionnels maritimes qui recherchent l'anguille sub-adulte et éventuellement d'autres espèces d'amphihalins. Cette catégorie de pêcheurs n'étant pas assujettis à un régime de licence de pêche pour exercer, leur dénombrement s'avère difficile et c'est pourquoi nous rapportons les seuls effectifs estimés pour la pêche de la civelle dans CASTELNAUD et al. (1994).

Les effectifs de pêcheurs non-professionnels fluviaux correspondent à des pêcheurs qui recherchent de façon avérée des espèces amphihalines dans les départements côtiers et de façon probable au moins l'anguille sub-adulte dans les autres départements (Figure 1). Dans les départements côtiers le nombre de pêcheurs et la variété des licences attribuées témoignent de l'importance de ces pêches. Par exemple dans le département de la Gironde qui concentre le plus grand nombre de pêcheurs non-professionnels fluviaux à pied ou en bateau, on dénombre en 1997 sur les seules zones mixtes de Garonne Dordogne - Isle, près de 200 pêcheurs avec une licence filet amateur de $60 \mathrm{~m}, 476$ pêcheurs avec une licence petite pêche amateur ou anguille amateur, 116 pêcheurs avec une licence tamis civelle amateur et près de 1400 pêcheurs avec une licence carrelet amateur.

Les 7536 pêcheurs non-professionnels fluviaux dénombrés au niveau national englobent les pêcheurs non-professionnels fluviaux de civelle dont l'effectif avait été estimé à 1250 en 1989 par CASTELNAUD et al. (1994).

Les pêcheurs de loisir aux lignes peuvent certainement pêcher à la fois le saumon et la truite de mer dans certains secteurs. Mais comme nous ne disposons pas d'information à ce sujet, nous considèrerons que la somme des effectifs des pêcheurs aux lignes de saumon et des pêcheurs aux lignes de truite de mer représente en 1997 l'effectif total officiel des pêcheurs aux lignes de salmonidés, soit 5004 . A ces pêcheurs aux lignes de salmonidés s'ajoutent les pêcheurs aux lignes d'alose en Dordogne, Garonne, Gaves d'Oloron et de Mauléon, Rhône et Ardèche et surtout les pêcheurs aux lignes d'anguille, dont le nombre est inconnu mais sans doute non négligeable. 


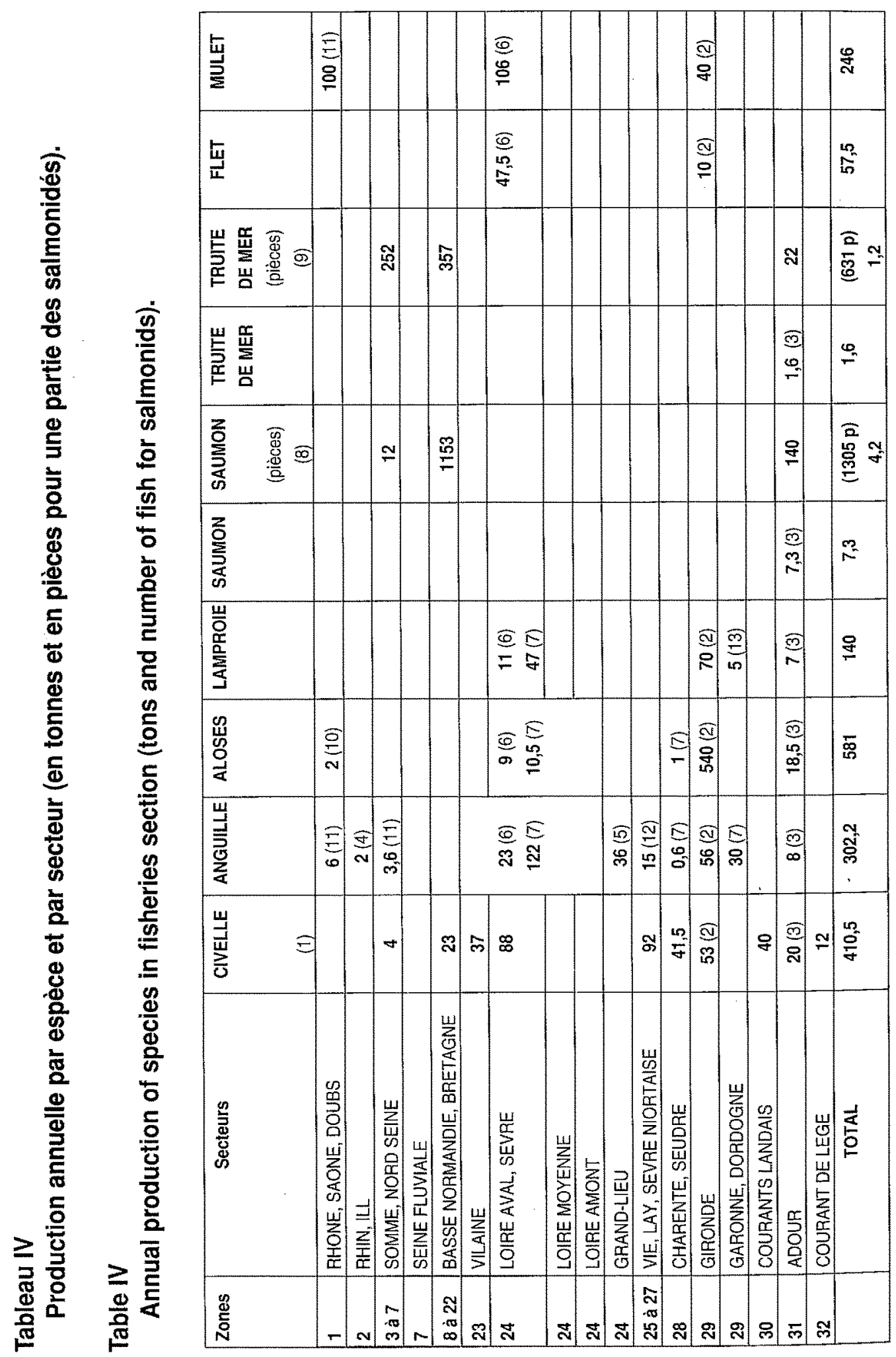

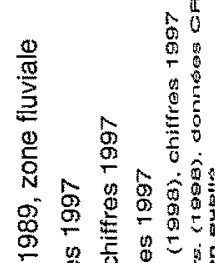

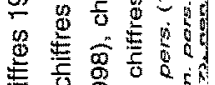

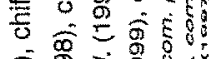

可要

$\pm \frac{1}{6} \overline{0}$

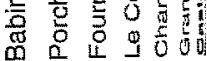

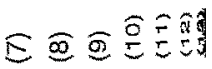

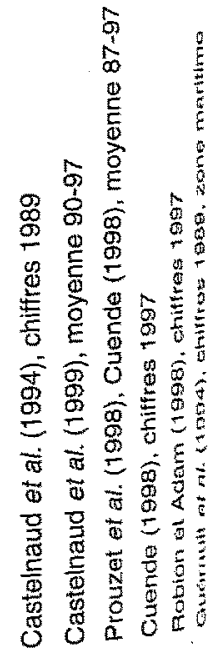

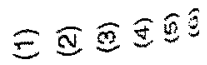




\section{Les productions}

Le Tableau IV présente les différentes données de production disponibles sur la période 1989 - 1997 pour les principales espèces d'amphihalins et ceci par secteur, selon le même découpage que pour les effectifs de pêcheurs au Tableau III. Pour chaque espèce et chaque secteur on a choisi la donnée de capture qui paraît la plus représentative : par exemple la capture moyenne pour la Gironde et pour l'Adour, ou bien la donnée de capture minimale de CASTELNAUD et al. (1994) pour la civelle du fait de la tendance décroissante observée au niveau national.

La production d'alose feinte a été rapportée pour la Charente et le Rhône dans la colonne Alose du Tableau IV du fait que les captures y ont été estimées (LECORRE, 1999 ; BABIN, 1993). Celle de Gironde, de loin la plus importante, peut varier de 20 à 50 tonnes. Elle n'est pas indiquée du fait que son suivi est très aléatoire (CASTELNAUD et al., 1999). Cette production est de toutes façons inférieure aux fluctuations annuelles autour de la moyenne de celle de l'alose vraie. La production de lamproie fluviatile limitée au bassin de la Garonne ne doit représenter que quelques centaines de kilogrammes.

On obtient les chiffres suivants de production en tonnage et en valeur pour l'année de référence 1997 :

- civelle : 410,5 tonnes,

410,5 millions de francs, (prix moyen $1000 \mathrm{~F} / \mathrm{kg}$ );

- anguille sub-adulte : 302 tonnes,

18,1 millions de francs, (prix moyen $60 \mathrm{~F} / \mathrm{kg}$ ) ;

- aloses : 581 tonnes,

8,7 millions de francs, (prix moyen $15 \mathrm{~F} / \mathrm{kg}$ ) ;

- lamproie marine : 140 tonnes,

15,4 millions de francs, (prix moyen $110 \mathrm{~F} / \mathrm{kg}$ ) ;

- saumon : 11,5 tonnes,

1,4 million de francs, (prix moyen $120 \mathrm{~F} / \mathrm{kg}$ ) ;

- truite de mer : 2,8 tonnes,

0,2 million de francs, (prix moyen $70 \mathrm{~F} / \mathrm{kg}$ ) ;

- mulet : 246 tonnes,

1,7 million de francs, (prix moyen $7 \mathrm{~F} / \mathrm{kg}$ ) ;

- flet : 57,5 tonnes,

5,7 millions de francs, (prix moyen $10 \mathrm{~F} / \mathrm{kg}$ ) ;

soit une production totale en poids de 1751,5 tonnes (arrondies à 1750 tonnes) pour une valeur de 461,7 millions de francs (arrondis à 462 millions de francs). La Figure 3 compare ces productions par espèces ou groupes d'espèces. 


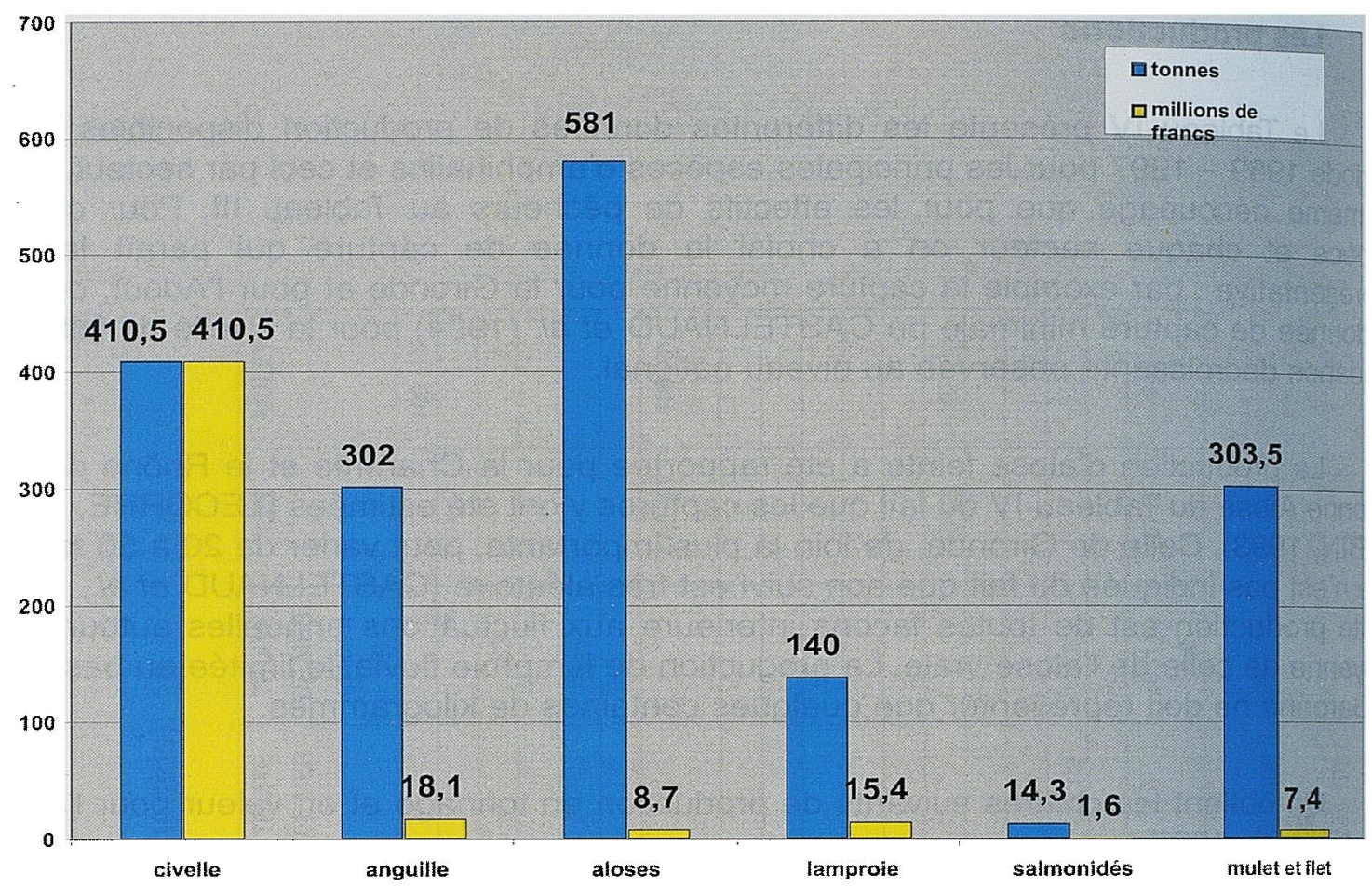

Figure 3

Production en tonnage et en valeur des principales espèces amphihalines dans les fleuves français.

\section{Figure 3}

\section{Production and value of the main migratory species fished in french rivers.}

On voit que le chiffre d'affaires pour certaines espèces est réalisé dans quelques bassins seulement : Gironde pour l'alose, Loire et Gironde pour la lamproie, Normandie, Bretagne, Adour pour les salmonidés. Les productions sont beaucoup plus disséminées pour l'anguille, tous stades confondus, ce qui est conforme à la répartition des pêches et des pêcheurs (Tableaux I et III ; Figures 1 et 2).

II faut noter que comme pour les salmonidés, la production des pêcheurs autres que professionnels a été prise en compte pour les autres espèces lorsque cela a été possible. Ceci est le cas pour la civelle dans l'ensemble des zones de pêche (CASTELNAUD ef al, 1994), pour l'alose vraie, l'anguille et la lamproie marine en Gironde (CASTELNAUD ef als, 1999). De ce fait, pour la civelle, les aloses et la lamproie marine, les chiffres représentent pratiquement toute la production nationale. Par contre, cela est moins sûr pour l'anguille sub-adulte qui peut être pêchée par de nombreux pêcheurs non-professionnels aux filets et aux engins et par des pêcheurs aux lignes.

Ceci est moins sûr aussi pour les salmonidés qui ont la particularité d'être spécialement recherchés par les pêcheurs aux lignes, mais aussi semble-t-il par des pêcheurs amateurs aux filets et aux engins. Comme pour les autres espèces, les estimations de captures sont entachées d'incertitudes, ce qui est confirmé par les taux de déclarations des captures de saumon indiqués par PORCHER (1998) et les taux de déclarations de captures de truite de mer discutés par FOURNEL et al. (1998). D'après cet auteur, sur la rivière atelier la Bresle, près de 80 truites de mer non-déclarées ont êtét capturées à la ligne en 1997 pour 37 déclarées et 250 à 300 truites de mer non-déclarées ont été capturées par les pêcheurs à pied au filet à proximité de l'embouchure ; les captures 
de la pêche professionnelle et non-professionnelle en bateau sont inconnues (FOURNEL et al. 1998). De telles captures (accidentelles ou dirigées) sont certainement réalisées par des pêcheurs aux filets et engins dans d'autres bassins, en Gironde-Garonne-Dordogne par exemple. Les captures réelles totales de salmonidés sont estimées à 4000 par EUZENAT (com. pers.).

Si l'on s'en tient aux chiffres de captures répertoriés, on constate que le poids des captures des pêcheurs aux lignes est évaluée à 4,2 tonnes pour le saumon et 1,2 tonne pour la truite de mer. Pour pouvoir estimer un chiffre d'affaires de la pêche des amphihalins, nous avons pris le parti de calculer un chiffre d'affaires correspondant à la production totale de salmonidés à partir du seul prix de vente existant. Ce choix peut être contesté. Certains défenseurs des salmonidés par exemple, arguent du fait que la valeur du poisson noble pêché à la ligne est nettement plus importante que celle occasionnée par la pêche professionnelle. Mais de toutes façons, même si le chiffre d'affaires correspondant aux salmonidés est sous-évalué, il ne représente qu'un faible pourcentage du chiffre d'affaires de la pêche de l'ensemble des espèces amphihalines qui est dominé par l'espèce anguille, spécialement le stade civelle qui en détermine à lui seul $89 \%$.

\section{Tableau V}

Comparaison en valeur de la civelle d'anguille aux autres espèces du Golfe de Gascogne en 1981, 1989 et 1997.

Table V

Comparison of the value of glass eel and others species in the Bay of Biscay in 1981,1989 and 1997.

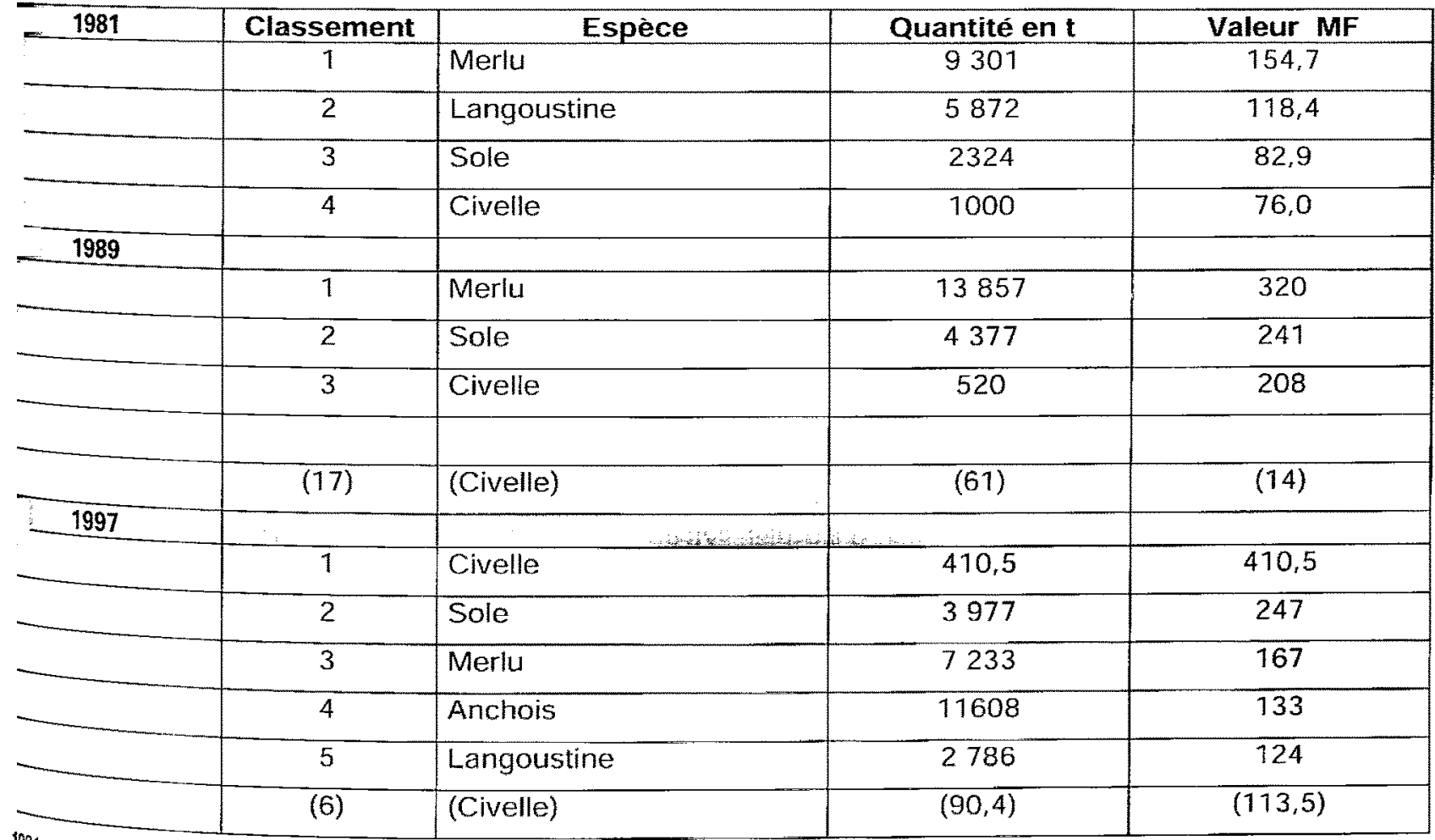

Données du rapport Technique ISTPM $n^{\circ} 3 b(1983)$.

Données de l'état statistique CRTS (GRANDPIERRE, com. pers.).

Données de l'état statistique CRTS (GRANDPIERRE, com. pers.).

Nouveau classement suite à la valeur corrigée par le Groupe Evaluation des stocks de lISTPM pour

(ivelle): Classement originel et valeur dans l'état statistique du CRTS (GRANDPIERRE, com. pers.). 
En 1981, la valeur corrigée de la civelle (ISTPM, 1983) essentiellement produite sur la façade atlantique (Tableau IV) plaçait celle-ci au $4^{e}$ rang des ressources du Golfe de Gascogne après le merlu, la langoustine et la sole (Tableau V) ; en 1989, avec 520 tonnes et une valeur de $208 \mathrm{MF}$, celle-ci remontait à la $3^{2}$ place, après le merlu et la sole. En 1997, avec une production estimée à 410,5 tonnes et une valeur associée de $410,5 \mathrm{MF}$, la civelle passe en tête avec une valeur 1,66 fois supérieure à celle de la sole. ll est à noter qu'en 1989, le CRTS, sur la base des déclarations de captures des marins-pêcheurs, plaçait la civelle en $17^{\circ}$ position et en 1997 , en $5^{\mathrm{e}}$ position.

Dans l'état statistique 1997, on s'aperçoit que la production de civelle déclarée est presque cinq fois inférieure à celle que nous avons estimée. En fait, les statistiques du CRTS prennent en compte uniquement la pêche des marins-pêcheurs ; les 90 tonnes déclarées sont donc à comparer à la production estimée des marins-pêcheurs uniquement, soit 260 tonnes, chiffre qui rejoint les évaluations récentes de 2 à 300 tonnes de la CIPE (GUERNALEC 1998, com. pers.). Le rapport entre production déclarée et production estimée n'est plus de 1 à 5 , mais de 1 à 3 . Nous ne pouvons connaître le taux de sous-déclaration pour chaque espèce mais on peut penser qu'il est beaucoup pils important pour la civelle que pour les autres espèces. II faudrait que ce taux soit dials moins $40 \%$ pour la sole afin qu'elle conserve sa $1^{\text {te }}$ place théorique.

L'anguille au stade sub-adulte, avec une production estimée de 302 tonnes et un chiffre d'affaires de $18,1 \mathrm{MF}$, remonte dans cet état statistique de la $46^{\mathrm{e}}$ place à la $17^{ \pm}$ place entre le céteau et le lieu jaune. Si l'on ajoute la valeur de cette production d'angulle sub-adulte à la valeur de la production de la civelle pour considérer l'espèce anguille tows stades confondus, on obtient $428,6 \mathrm{MF}$, ce qui conforte sa suprématie par rapport aux ressources halieutiques du golfe de Gascogne et à l'ensemble des espèces amphithaliniss puisqu'elle représente alors $93 \%$ du chiffre d'affaires total.

Mais cette suprématie qui repose sur le chiffre d'affaires de la pêche d'un alevin est aléatoire et par voie de conséquence la rentabilité des entreprises de pâthe professionnelle basée essentiellement sur les espèces amphihalines, pourrait être mise cause. En effet, la part de la pêche de la civelle représente en 1997 plus de $50 \%$ chiffre d'affaires total pour les pêcheurs professionnels fluviaux en Gironde et en Adtaur d'après CHAMPION (1999), plus de $70 \%$ du chiffre d'affaires des marins-pêcheurs th Adour d'après PROUZET et al., (1998) et une part certainement prépondérante du chililie d'affaires des quelques 700 marins-pêcheurs qui font la saison de civelle dans les embouchures et les estuaires et qui pêchent les espèces marines pour le reste du temps.

Il faut aussi remarquer que la mobilisation de la majorité des pêcheurs professionnels maritimes et fluviaux sur la pêche de la civelle permet aux autres d"awairle terrain plus libre pour pratiquer d'autres types de pèche comme celle de la lamproie marine, de l'anguille sub-adulte, de la crevette et de rentabiliser leur entreprise à partir ces pêches, ce qu'ils ne pourraient faire si la concurrence devenait importante, du failt diune réduction de la pêche de la civelle et d'une reconversion des pêcheurs.

\section{CONCLUSION}

Nous avons rếalisé un bilan socio économique de la pêche des amphihalines dans les fleuves français à la fin des annés 1990, plus exactememintur. l'année de référence 1997 avec des données éparses couvrant la période 1989 - 11998

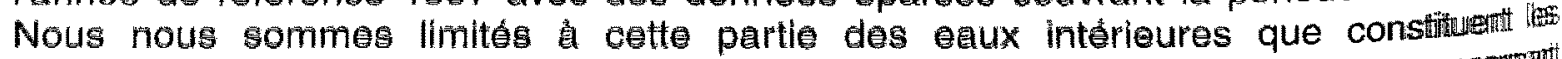
hydrosystèmes fluviaux par souci de cohếrence et du fẩ que les informations concentmint les lagunes méditerranéennes sont diffleiles d'acess ou font défaut pendant cette partine 
Dans le chiffre d'affaires de la filière pêche des espèces amphihalines nous avons montré la place secondaire des salmonidés et la place stratégique de l'espèce anguille, spécialement du stade civelle dont la valeur économique est hypertrophiée par les prix pratiqués depuis quelques années.

Assurément, ce sont d'abord les emplois et les revenus que génèrent les activités de pêche commerciale et de loisir qui déterminent le plus, actuellement, l'importance sociale et économique des espèces amphihalines. C'est d'ailleurs sur la valorisation actuelle ou potentielle de ces espèces par la pêche que l'on s'appuie pour justifier les plans et actions de restauration et pour s'opposer à l'implantation de projets industriels ou obtenir une limitation des impacts. Mais cette valorisation est sujette à controverse ; des conflits d'intérêt existent et des problèmes de partage de ces ressources naturelles renouvelables se posent.

Du fait des particularités, de l'originalité et de la complexité de leurs cycles biologiques, de leurs qualités esthétiques ou de leur étrangeté, de leurs qualités gastronomiques, les espèces amphihalines ont une valeur symbolique, culturelle, patrimoniale qui s'ajoute à leur intérêt social et économique.

La gestion de ces espèces peut permettre d'envisager un redéploiement de l'activité de la pêche professionnelle, cependant qu'elle sera limitée par le principe de précaution, par une redistribution entre les différents utilisateurs et de nouvelles affectations des stocks. La restauration possible et la pêche potentielle dans le futur d'une espèce amphihaline supplémentaire, l'esturgeon Acipenser sturio donne à la France une responsabilité accrue dans la bio-conservation au niveau européen ; la France est déjà, en effet, le seul pays d'Europe de l'Ouest à détenir des populations d'espèces amphihalines autres que l'anguille ef les salmonidés à un niveau suffisant pour permettre une exploitation par pêche.

Les suivis statistiques officiels, lancés au niveau national, devraient permettre à

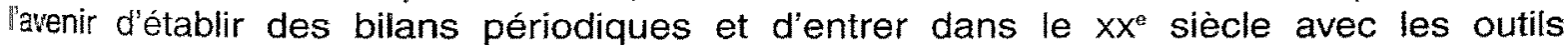
indispensables à la gestion des espèces amphihalines. On ne peut préjuger de leur efficacité, qui sera à la mesure des moyens consentis et des méthodes appliquées, mais malgré l'optimisme indispensable affiché par ses promoteurs, il faudra sans doute que passe une génération de pêcheurs pour obtenir des résultats fiables, réalistes et représentatifs, compte tenu d'une part, du travail à réaliser pour obtenir une bonne couverture de la peche professionnelle et non-professionnelle et d'autre part du climat de concurrence et de méfiance qui règne à cause de l'état apparent des stocks et des temps de réponse des espèces aux actions de restauration et de gestion, des restrictions administratives et réglementaires ainsi que des enjeux financiers.

\section{REMERCIEMENTS}

Je suis très redevable a David BARBIER of à Philippe CAMOIN pour leur aide dans la réalisation des Tableaux, Figures et dans la mise en forme du texte.

\section{BIBLIOGRAPHIE}

ALLARDI J., KEITH P., (coord), 1991. Atlas préliminaire des poissons d'eau douce de France. Coll. Patrimoines Naturelg, vol. 10. Secrétariat Faune-Flore (MNHN), CSP, Min. Env., CEMAGREF, Paris, 23 p.

AMONYME, 1987. Les captures de polssons migrateurs dans les ostuaires français depuis le milieu du $\times \times$ siocle. Rapport INRA Ronnes/ENSA Ronnes, $48 p+$ annexes. 
AUBRUN L., 1986. Inventaire de l'exploitation de l'anguille sur le littoral de la Bretagne. Laboratoire de Biologie Halieutique, Rennes, Les publications du département d'Halieutique $n^{\circ}$ 1. $107 \mathrm{p}$.

BABIN D. 1993. Contribution à l'étude d'une ressource naturelle renouvelable : la pêche professionnelle en eau douce en France. Thèse $3^{e}$ cycle CEMAGREF/Univ. Rennes II, $300 \mathrm{p}$.

CASTELNAUD G., BABIN D., 1992. La pêche professionnelle fluviale et lacustre en France : enquête au fil de l'eau. CEMAGREF Dicova, Antony, Etudes Ressources en Eau n ${ }^{\circ} 5.291$ p.

CAstelnaud G., Guerault D., Desaunay Y., EliE P., 1994. Production et abondance de la civelle en France au début des années 90. Bull. Fr. Pêche Piscic, $n^{\circ} 335,263-287$.

CASTELNAUD G., ROCHARD E., GAZEAU C ., 1999. Surveillance halieutique de l'estuaire de la Gironde - Suivi des captures 1997 - Etude de la faune circulante 1998. Etude CEMAGREF, groupement de Bordeaux, $n^{\circ} 41.172 \mathrm{p}$.

CASTELNAUD G., DE VERDILHAC Ph., 1982. Etat de la production des pêches d'estuaires : Importance de cette évaluation et méthodologie. Acte du colloque international des Entretiens Ecologiques de Dijon, p. 98-107.

CHAMPION L., 1999. Etude socio-économique des pêches professionnelles continentales. Rapport CNAPPED/CSP/Min. Agriculture et de la Pêche/LEN-CORRAIL Nantes, 92 p. + annexes.

CHANCEREL F., 1994. La répartition de l'anguille en France. Bull. Fr. Pêche et Piscic. (1994) $335: 289-294$.

CUENDE F.X., 1998. Etat général des pêches continentales et estuariennes : présentation statistique in : Luce Gagnaire coord. Des pêcheurs professionnels au centre de l'Europe, actes des $5^{\mathrm{e}}$ assises nationales de la pêche professionnelle continentale et estuarienne, Thonon-les-Bains, 1998, p. 5-8 et annexes.

DESAUNAY Y., AUBRUN L., 1988. Description des pêcheries d'anguille (Anguilla anguilla) sur le littoral français de la Manche et de l'Atlantique. Cons. Int. Explor. Mer, C.M. 1988, M:25, $15 \mathrm{p}$.

DILL W.A., 1993. Inland fisheries in Europe FAO-EIFAC technical paper, 52, suppl. 281 p.

ELIE P., 1986. Les productions halieutiques et aquacoles dans les eaux continentales françaises. Symposium FAO-CECPI, Bordeaux, mai et juin 1986, $7 \mathrm{p}$.

ELIE P., 1992. L'hydrobiologie des estuaires. Colloque national estuaires et deltas : des milieux menacés, Bègles, 25 juin 1992. in : Estuaires et deltas : des milieux menacé, 9 p. Agence de l'Eau Adour-Garonne - IFREMER - Min. Environnement.

ELIE P., 1993. Les grandes espèces migratrices du bassin versant Gironde-GaronneDordogne : biologie, importance économique et évolution de l'exploitation. Pour un retour des poissons migrateurs, 9-10-11 décembre 1993, Toulouse. in : Pour un retour des poissons migrateurs: Adour-Charente-Dordogne-Garonne, 9 p. Agence de l'eau Adour-Garonne, CSP, CNRS.

ELIE P., RIGAUD C., 1984. Etude de la population d'anguilles de l'estuaire et du bassin versant de la Vilaine : pêche-biologie-écologie, examen particulier de l'impact du barrage d'Arzal sur la migration anadrome (civelles) : t.1. CEMAGREF, Bordeaux. Fac de Rennes/URFDAAPP, $174 \mathrm{p}$.

FOURNEL F., FAYARD J.L., EUZENAT G., RICHARD A., PORCHER J.P., HOUSSAIS P., 1998. La truite de mer en France année1997. Le suivi des captures en France. La Pêche de la truite de mer en Normandie - Artois - Picardie. Eléments de connaissance des stocks en France. Suivi des populations et des captures rivière atelier Bresle. Rapport CSP, 47 p. + annexes. 
GUERAULT D., DESAUNAY Y., BEILLOIS P., 1994. La pêche professionnelle des poissons migrateurs dans l'estuaire de la Loire en 1989. Repères Océan, IFREMER, $n^{\circ} 6,3-28$.

IS.T.P.M., 1983. Réflexions sur les besoins en matière de recherche sur les pêches et les cultures marines. Rapports des groupes régionaux ou sectoriels. Partie golfe de Gascogne. Rapp. Technique $n^{\circ} 3 b, 16 \mathrm{p}$.

KEITH P., 1998. Evolution des peuplements ichtyologiques de France et stratégies de conservation. Thèse de doctorat. Spécialité Biologie. Université de Rennes I. 239 p.

LE CORRE M., 1999. Eco-biologie et génétique des populations d'aloses (Alosa fallax spp) de la Méditerranée française. Thèse de doctorat. Spécialité halieutique, école nationale supérieure agronomique de Rennes. $189 \mathrm{p}$.

PORCHER J.P., 1998. Le saumon atlantique en France en 1997. Captures pour les pêcheurs amateurs et professionnels en eau douce, éléments de connaissance et de gestion des stocks. Rapport CSP, $45 \mathrm{p}$.

PROUZET P., MARTINET J.P., DECASAMAJOR M.N., POUSSIN L., 1998. Rapport sur la pêche des marins-pêcheurs dans l'estuaire de l'Adour en 1997. IFREMER/INRA, St-Pée., 44 p.

ROBION D., ADAM G., 1998. Les pêcheurs professionnels du lac de Grand-Lieu. Poster. $5^{\text {es }}$ Assises de la Pêche Professionnelle Continentale et Estuarienne à Thonon-lesBains, 4 au 7 nov. 1998. 